\title{
FACTORING FUNCTIONS ON CARTESIAN PRODUCTS
}

\author{
BY \\ N. NOBLE AND MILTON ULMER( $\left.{ }^{1}\right)$
}

\begin{abstract}
A function on a product space is said to depend on countably many coordinates if it can be written as a function defined on some countable subproduct composed with the projection onto that subproduct. It is shown, for $X$ a completely regular Hausdorff space having uncountably many nontrivial factors, that each continuous real-valued function on $X$ depends on countably many coordinates if and only if $X$ is pseudo- $\aleph_{1}$-compact. It is also shown that a product space is pseudo- $\boldsymbol{\aleph}_{1}$ compact if and only if each of its finite subproducts is. (This fact derives from a more general theorem which also shows, for example, that a product satisfies the countable chain condition if and only if each of its finite subproducts does.) All of these results are generalized in various ways.
\end{abstract}

Introduction. A function $f$ on a product space $X=\prod_{\alpha \in A} X_{\alpha}$ is said to depend on countably many coordinates if, for some countable subset $C$ of $A$, and some function $g$ on $\prod_{\alpha \in C} X_{\alpha}, f$ is equal to $g \circ \Pi_{C}$, where $\Pi_{C}$ is the projection from $X$ to $\prod_{\alpha \in C} X_{\alpha}$. The problem of determining conditions on a product space $X$ and a range space $Y$ under which each continuous function from $X$ to $Y$ will depend on countably many coordinates has developed a considerable literature which is very nicely summarized in [E], with one exception. That exception is the recent paper [V] by Vidossich in which it is shown that any uniformly continuous function from a subspace $Z$ of a product space into a metric space can be factored as the composition of a projection onto some countable subproduct with a function uniformly continuous on the projection of $Z$. As applied to real-valued functions, all of these results, except that of Vidossich, are generalized by our main theorem, that if $X$ is pseudo- $\boldsymbol{\aleph}_{1}$-compact, then each continuous real-valued function on $X$ depends upon countably many coordinates. If $X$ is the product of uncountably many nontrivial completely regular Hausdorff spaces, then the converse also holds. (For any infinite cardinal $\boldsymbol{\aleph}$, a space is said to be pseudo- $\boldsymbol{\aleph}$-compact provided each locally finite family of open subsets has cardinality less than $\aleph$; pseudocompact spaces, Lindelöf

Received by the editors August 27, 1970 and, in revised form, April 21, 1971.

AMS 1969 subject classifications. Primary 5425, 5428, 5440, 5453, 5460; Secondary 4160, 4625.

Key words and phrases. Functions depending on countably many coordinates, pseudo-ncompact spaces, countable chain condition, realcompactifications of infinite products, infinite products.

(1) This paper contains parts of the first two chapters of the author's doctoral dissertation $\left[\mathrm{U}_{2}\right]$ which was written under the guidance of Professor W. W. Comfort. Work on this paper was partially supported by the National Science Foundation under grants NSF-GP-8357 and NSF-GP-18825. 
spaces and separable spaces are all pseudo- $\boldsymbol{\aleph}_{1}$-compact.) This and some related results are established in $\S 2$. In the first section we prove some product theorems for the properties which we will be considering, showing in particular that a product space is pseudo- $\boldsymbol{\aleph}_{1}$-compact or satisfies the countable chain condition if and only if each of its finite subproducts does. We give generalizations of our results to higher cardinals in the final section.

For any set $S$, we denote by card $(S)$ the cardinality of $S$. Cardinal numbers are taken to be the set of their ordinal predecessors, and if $\boldsymbol{N}$ is an infinite cardinal, we denote by cof $(\boldsymbol{\aleph})$ the cofinality number of $\boldsymbol{\aleph}$.

We are grateful to W. W. Comfort on two counts: Firstly, his many suggestions and criticisms have greatly improved both the form and content of this paper, and secondly, he provides a constant source of inspiration to both authors.

1. Some product theorems. In this section we give product theorems concerning some of the properties which will be used in the next section.

1.1. Lemma. Let $\mathscr{F}$ be a family of finite sets and suppose card $(\mathscr{F})=\mathfrak{n}$, where $\mathfrak{n}$ is uncountable.

(i) If $\mathfrak{n}$ is regular, then there exist a finite set $F$ and an $\mathfrak{n}$-fold subfamily $\mathscr{F}^{\prime}$ of $\mathscr{F}$ such that, for any two members $G$ and $H$ of $\mathscr{F}^{\prime}$, we have $G \cap H=F$.

(ii) If $\mathfrak{n}$ is singular, then there exist a set $B$, with card $(B) \leqq \operatorname{cof}(\mathfrak{n})$, and an $\mathfrak{n}$-fold subfamily $\mathscr{F}^{\prime}$ of $\mathscr{F}$ such that, for any two members $G$ and $H$ of $\mathscr{F}^{\prime}$, we have $G \cap H \subset B$.

Proof. Suppose first that $\mathfrak{n}$ is regular. Since $\mathfrak{n}$ is uncountable, there must exist an integer $m$ and an $\mathfrak{n}$-fold subfamily $\mathscr{F}_{m}$ of $\mathscr{F}$ such that each member of $\mathscr{F}_{m}$ has precisely $m$ elements.

For each finite set $K$, let $\mathscr{F}(K) \subset \mathscr{F}_{m}$ be maximal with respect to the property that for any two members $G$ and $H$ of $\mathscr{F}(K)$ we have $G \cap H=K$. Let $F$ be maximal with respect to the property that $F$ is contained in each member of some $\mathfrak{n}$-fold subfamily of $\mathscr{F}_{m}$. It is easy to see that card $(\mathscr{F}(F))=\mathfrak{n}$. By setting $\mathscr{F}^{\prime}=\mathscr{F}(F)$, we have proved part (i).

If $\mathfrak{n}$ is singular, write $\mathfrak{n}=\sup _{\gamma \in \mathfrak{m}} \mathfrak{n}_{\gamma}$ where $\tau<\gamma$ gives us $\mathfrak{n}_{\tau}<\mathfrak{n}_{\gamma}$, and each $\mathfrak{n}_{\gamma}$ is regular. For each ordinal $\gamma \in \mathfrak{m}$, find a finite set $F_{\gamma}$ and an $\mathfrak{n}_{\gamma}$-fold subfamily $\mathscr{F}_{\gamma}$ of $\mathscr{F}$ as guaranteed by part (i). Set $B=\bigcup_{\gamma \in \mathfrak{m}} F_{\gamma}$. Clearly card $(B) \leqq \mathfrak{m}$. We will choose the desired subfamily $\mathscr{F}^{\prime}$ from $\bigcup_{\gamma \in \mathfrak{m}} \mathscr{F}_{\gamma}$.

We may assume $\mathfrak{n}_{\gamma}>\mathfrak{m}$ for all $\gamma \in \mathfrak{m}$. We define

$$
\mathscr{F}_{\gamma}^{\prime}=\mathscr{F}_{\gamma}-\bigcup_{\tau<\gamma} \bigcup_{G \in \mathscr{F}_{\tau}}\left\{H \in \mathscr{F}_{\gamma}: H \cap G \notin B\right\} .
$$

Since $H \cap K \subset B$ for $H$ and $K$ in $\mathscr{F}_{y}$, and since $G$ is finite, each family

$$
\left\{H \in \mathscr{F}_{\gamma}: H \cap G \notin B\right\}
$$

is finite. Consequently, since card $\left(\mathscr{F}_{\tau}\right)=\mathfrak{n}_{\tau}<\mathfrak{n}_{\gamma}$ and $\mathfrak{n}_{\gamma}$ is regular, we see that $\operatorname{card}\left(\mathscr{F}_{\gamma}^{\prime}\right)=\mathfrak{n}_{\gamma}$. Thus, if we set $\mathscr{F}^{\prime}=\bigcup_{\gamma \in \mathfrak{m}} \mathscr{F}_{\gamma}^{\prime}$, we have the desired subfamily and the lemma is proved. 
Lemma 1.1(i) is not new; it has been proven by N. Šanin $\left[S_{3}\right]$ as well as by S. Mazur $\left[\mathrm{M}_{3}\right]$. Other forms and proofs of it can be found in [D], [ER], and $\left[\mathrm{M}_{4}\right]$. The second part of the lemma seems to have been overlooked by these authors.

Let $\phi$ be a statement about families of subsets (or subspaces) of a topological space. We call such a statement saturated provided

(a) if $\mathscr{U}$ has $\phi$ and $\mathscr{U}^{\prime} \supset \mathscr{U}$, then $\mathscr{U}^{\prime}$ has $\phi$;

(b) if $\left\{U \times V_{0}: U \in \mathscr{U}\right\}$ has $\phi$, then $\mathscr{U}$ has $\phi$;

(c) if $\left\{U_{y}: \gamma \in \Gamma\right\}$ has $\phi$ and if, for each index $\gamma \in \Gamma, V_{\gamma}$ is a nonempty subset of $X_{\gamma}$, then the family $\left\{U_{\gamma} \times V_{\gamma} \times \prod_{\alpha \in \Gamma-\{\gamma\}} X_{\alpha}: \gamma \in \Gamma\right\}$ has $\phi$.

Recall that a family of sets is said to have the $\mathfrak{n}$-intersection property provided each subfamily of cardinality at most $\mathfrak{n}$ has nonempty intersection. A point is a cluster point of a family of sets if each neighborhood of the point meets infinitely many members of the family; it is an adherent point if it is in the closure of each member of the family.

1.2. ExAmples. For any cardinal numbers $\mathfrak{m}$ and $\mathfrak{m}^{\prime}$, each of the following statements is saturated:

(i) The family $\mathscr{U}$ contains an $\mathfrak{m}$-fold subfamily with the $\mathfrak{m}^{\prime}$-intersection property.

(ii) The family $\mathscr{U}$ contains an $\mathfrak{m}$-fold subfamily each $\mathfrak{m}^{\prime}$-fold subfamily of which has an adherent point.

(iii) The family $\mathscr{U}$ contains an $\mathfrak{m}$-fold subfamily each $\mathfrak{m}^{\prime}$-fold subfamily of which has a cluster point.

1.3. THEOREM. Let $\phi$ be a saturated statement, let $\mathfrak{n}$ be an uncountable cardinal number, and let $P(\phi, \mathfrak{n})$ be a topological property which can be formulated as: Each $\mathfrak{n}$-fold family of nonempty basic open sets (repetitions allowed) satisfies $\phi$.

(i) If $\mathfrak{n}$ is regular, then a product space has $P(\phi, \mathfrak{n})$ if and only if each finite subproduct does.

(ii) If $\mathfrak{n}$ is singular, and $\mathbb{N}=\operatorname{cof}(\mathfrak{n})$, then a product space has $P(\phi, \mathfrak{n})$ if and only if each subproduct of $\boldsymbol{\aleph}$ factors does.

Proof. Suppose $X=\prod_{\alpha \in A} X_{\alpha}$ has $P(\phi, \mathfrak{n})$, and $B$ is any nonempty subset of $A$. Let $\mathscr{U}$ be an $\mathfrak{n}$-fold family of nonempty basic open subsets of the subproduct $\prod_{\alpha \in B} X_{\alpha}$. Since $\left\{U \times \prod_{\alpha \in A-B} X_{\alpha}: U \in \mathscr{U}\right\}$ is an $\mathfrak{n}$-fold family of nonempty basic open subsets of $X$, this family must have $\phi$. But then, using (b), $\mathscr{U}$ must have $\phi$. Thus the "only if" portions of both (i) and (ii) are established.

For the proof of the converses, we must introduce some notation. For each nonempty basic open subset $U$ of $X$, let $R(U)$ denote the finite set of coordinates on which the projection of $U$ is not the entire factor.

Now, let $\mathscr{U}$ be any $\mathfrak{n}$-fold family of nonempty basic open subsets of $X$. Under the appropriate assumptions of (i) or (ii) on subproducts of $X$, we will show that $\mathscr{U}$ satisfies $\phi$.

Applying Lemma 1.1 to the family $\{R(U): U \in \mathscr{U}\}$, we can choose a nonempty set $B \subset A$ and an $\mathfrak{n}$-fold subfamily $\mathscr{U}^{\prime}$ of $\mathscr{U}$ such that $\prod_{\alpha \in B} X_{\alpha}$ has $P(\phi, \mathfrak{n})$ and such 
that for any two sets $U$ and $V$ in $\mathscr{U}^{\prime}$ we have $R(U) \cap R(V) \subset B$. Since $\prod_{\alpha \in B} X_{\alpha}$ has $P(\phi, \mathfrak{n})$, we know that $\Pi_{B}\left(\mathscr{U}^{\prime}\right)$ has $\phi$. It follows by (c) that $\mathscr{U}^{\prime}$ has $\phi$, and hence, using (a), we see that the original family $\mathscr{U}$ has $\phi$.

Let $\phi$ be as in part (i) of Example 1.2. For $\mathfrak{n}=\mathfrak{m}=\mathfrak{m}^{\prime}$, the property $P(\phi, \mathfrak{n})$ is called caliber $\mathfrak{n}$, and for $\mathfrak{m}=\boldsymbol{\aleph}_{1}$ and $\mathfrak{m}^{\prime}=2$, spaces with $P\left(\phi, \boldsymbol{\aleph}_{1}\right)$ are said to have property $(K)$. It was shown by Sanin in $\left[S_{3}\right]$ that, for $\mathfrak{n}$ regular and uncountable, caliber $\mathfrak{n}$ is preserved by products, and by Marczewski in $\left[M_{1}\right]$ that property $(K)$ is preserved by products. Theorem 1.3 yields the nontrivial part of the proofs of these theorems. For $\mathfrak{m}=\mathfrak{m}^{\prime}=2$, spaces with $P(\phi, \mathfrak{n})$ are said to satisfy the $\mathfrak{n}$-chain condition (for $\mathfrak{n}=\boldsymbol{\aleph}_{1}$, they are said to satisfy the countable chain condition) and for this case Theorem 1.3 yields some new results which we state below. For further information concerning these properties, see [E].

1.4. COROLlaRY. (i) If $\mathfrak{n}$ is regular and uncountable, then a product space satisfies the $\mathfrak{n}$-chain condition if and only if each finite subproduct does.

(ii) If $\mathfrak{n}>\aleph_{0}$ is singular, and $\mathbb{N}=\operatorname{cof}(\mathfrak{n})$, then a product space satisfies the $\mathfrak{n}$-chain condition if and only if each subproduct of $\boldsymbol{\aleph}$ factors does.

This result for the countable chain condition is apparently not new. Franklin Tall informs us that it has been known among Wisconsin set-theorists and topologists for some time.

1.5. Corollary. (i) If $\mathfrak{n}$ is regular and uncountable, then a product space is pseudo-n-compact if and only if each finite subproduct is.

(ii) If $\mathfrak{n}>\boldsymbol{\aleph}_{0}$ is singular, and $\boldsymbol{\aleph}=\operatorname{cof}(\mathfrak{n})$, then a product space is pseudò- $\mathfrak{n}-c o m p a c t$ if and only if each subproduct of $\boldsymbol{\aleph}$ factors is.

Proof. For $\phi$ as in part (iii) of Example 1.2 and $\mathfrak{n}=\mathfrak{m}=\mathfrak{m}^{\prime}$, the property $P(\phi, \mathfrak{n})$ is pseudo-n-compactness.

We will give examples below to show that various parts of Corollaries 1.4 and 1.5 (and hence various parts of Theorem 1.3) cannot be improved. For comparison, recall that, by results in $[G],\left[C_{1}\right]$, and $[F]$, a product space is psuedocompact (i.e. pseudo- $\boldsymbol{\aleph}_{0}$-compact) if each of its countable subproducts is. However, for each cardinal $\mathfrak{n}$ with $2 \leqq \mathfrak{n} \leqq \boldsymbol{\aleph}_{0}$, there exists a space $Y$ for which $Y^{\mathfrak{n}}$ is not pseudocompact even though each smaller product is. Our first example shows that "finite" in part (i) of Corollary 1.5 cannot be improved.

1.6. EXAMPLE. For each infinite cardinal $\aleph$ and each integer $n$ there exists a space $Y$ such that $Y^{n}$ is pseudocompact but $Y^{n+1}$ is not pseudo- $\boldsymbol{\aleph}$-compact.

Proof. This is a straightforward adaptation of the methods used in $\left[\mathrm{C}_{1}\right]$ and $[\mathrm{F}]$.

A similar example for the countable chain condition is impossible since it has been shown that the question of whether the countable chain condition is preserved by finite products is independent of Zermelo-Fraenkel set theory even with the axiom of choice adjoined. For a discussion of this see $\left[\mathrm{C}_{2}\right]$. 
Our final example will show that, in both corollaries, regularity in (i) cannot be weakened, and as a statement about all singular uncountable cardinals (ii) cannot be improved.

1.7. EXAmple. For each singular cardinal $\boldsymbol{\aleph}$ there exists a product space $Y$ such that $Y$ fails to be pseudo- $\boldsymbol{\aleph}$-compact, while each subproduct of fewer than cof $(\boldsymbol{N})$ factors satisfies the $\boldsymbol{K}$-chain condition.

Proof. Since $\aleph$ is singular there exist sets $A$ and, for each $\alpha$ in $A, Y_{\alpha}$, each of cardinality less than $\aleph$, such that $\bigcup_{\alpha \in A} Y_{\alpha}$ has cardinality $\aleph$. Giving each set the discrete topology, we set $Y=A \times \prod_{\alpha \in A} Y_{\alpha}$. Notice that each finite subproduct of $Y$ has cardinality less than $\boldsymbol{\aleph}$. Nevertheless, $Y$ is not pseudo- $\boldsymbol{\aleph}$-compact since sets of the form $\{\beta\} \times\{y\} \times \prod_{\alpha \neq \beta} Y_{\alpha}$ for $\beta$ in $A$ and $y$ in $Y_{\beta}$ form an $\boldsymbol{N}$-fold family of pairwise disjoint open sets which cover $Y$.

Let $B \subset A$ be any set of cardinality less than cof $(\boldsymbol{\aleph})$. To see that $\prod_{\alpha \in B} Y_{\alpha}$ has the $\boldsymbol{\aleph}$-chain condition, notice that there must exist some regular cardinal $\boldsymbol{\aleph}^{\prime}<\boldsymbol{\aleph}$ such that for each $\alpha \in B$ we have card $\left(Y_{\alpha}\right)<\boldsymbol{N}^{\prime}$. Thus each finite subproduct of $\prod_{\alpha \in B} Y_{\alpha}$ has the $\boldsymbol{N}^{\prime}$-chain condition. Now apply Corollary 1.4(i) to obtain that $\prod_{\alpha \in B} Y_{\alpha}$ has the $\boldsymbol{N}^{\prime}$-chain condition and hence also the $\boldsymbol{\aleph}$-chain condition.

Let us note that Theorem 1.3 can be generalized in several ways. First of all, it has obvious analogues for product sets topologized with the $\mathfrak{n}$-topology (where $\mathfrak{n}$-fold products of open sets form a basis). The needed analogue of Lemma 1.1 is provided, for example, in [D]. However, the results for these topologies are not as precise as those above because cardinal arithmetic plays a larger role.

Secondly, the proof of Theorem 1.3 can be adapted to conclude that a subspace $Z$ of the product space has $P(\phi, \mathfrak{n})$, where $P(\phi, \mathfrak{n})$ now means: For each $\mathfrak{n}$-fold family $\mathscr{U}$ of nonempty basic open subsets of the product, $\{U \cap Z: U \in \mathscr{U}\}$ has $\phi$. Of course, the hypotheses on $\phi$ must be modified: One needs to know that the traces of the various families on $Z$ will have $\phi$.

For a final generalization of Theorem 1.3 , note that the only property of basic open sets which is actually used is that they depend on finitely many coordinates, so such a set $U$ is completely known if $\Pi_{F}(U)$ is known, where $F=R(U)$ is finite. Thus the property $P(\phi, \mathfrak{n})$ in the statement of Theorem 1.3 can be replaced by corresponding properties formulated in terms of other families of "finitely determined" subsets. Furthermore, the set "determined" by $\Pi_{F}(U)$ need not be $\Pi_{F}^{-1}\left(\Pi_{F}(U)\right)$-it could for example be $\Pi_{F}(U) \times \Pi_{A-F}(Z)$ for some fixed subspace $Z$ of $\prod_{\alpha \in A} X_{\alpha}$. Of course, (c) in the definition of saturated should, in that case, be reworded to say that the family of subsets of $Y \times \prod_{\gamma \in \Gamma} X_{\gamma}$ "determined" by $\left\{U_{\gamma} \times V_{\gamma}: \gamma \in \Gamma\right\}$ has $\phi$ whenever $\left\{U_{\gamma} \subset Y_{\gamma}: \gamma \in \Gamma\right\}$ does.

As an example of some intrinsic interest, take as our finitely determined family the points of a $\Sigma_{0}$-subspace of a product. (Recall that a $\Sigma_{0}$-subspace of a product is a subspace consisting of all points which differ from a fixed point $p$ on at most finitely many coordinates; this subspace is denoted as $\Sigma_{0}(p)$. Of course, if $X$ is a product of groups, the $\Sigma_{0}$-subspace containing the identity is the direct sum of the 
factors.) Adapting Theorem 1.3 we see, for $\mathfrak{n}$ regular, that $\Sigma_{0}$-subspaces are $\mathfrak{n}-\mathfrak{n}$ compact (each $\mathfrak{n}$-fold open cover has a subcover of cardinality less than $\mathfrak{n}$ ) if and only if each finite subproduct is (since a space is $\mathfrak{n}-\mathfrak{n}$ compact if and only if each subset of cardinality $\mathfrak{n}$ has an accumulation point $x$ each neighborhood of which meets $\mathfrak{n}$ of the points of the subset). The corresponding result for the Lindelöf property follows; this was observed in [E] by essentially the same proof.

2. Functions which depend on countably many coordinates. Recall that a function on a product space is said to depend on countably many coordinates if it can be written as the composition of some function on a countable subproduct together with the projection onto that subproduct. We consider the problem: Under what conditions on a product space $X$ and a range space $Z$ will each continuous function from $X$ to $Z$ depend on countably many coordinates? For a thorough history of the study of this problem the reader is referred to [E]; here we will only describe the current state of its solution. In the direction of greatest generality for $Z$, Miščenko shows in $\left[\mathrm{M}_{5}\right]$ that a product space $X$ has the property that each continuous function from $X$ to $Z$ depends on countably many coordinates, for each $T_{2}$-space $Z$ in which each point is a $G_{\delta}$, if and only if $X$ has caliber $\aleph_{1}$. Strengthening the hypotheses on $Z$ slightly, Engelking shows in [E] that if each finite subproduct of $X$ is Lindelöf and the $T_{2}$-space $Z$ has $G_{\delta}$-diagonal (i.e., if the diagonal of $Z \times Z$ is a $G_{\delta}$ ) then each continuous function from $X$ to $Z$ depends on countably many coordinates. Actually, Engelking proves the stronger result:

2.1. Proposition. Let $Z$ be a $T_{2}$-space with $G_{\delta}$-diagonal. Let $X=\prod_{\alpha \in A} X_{\alpha}$, and for each $\alpha \in A$ let $Y_{\alpha}$ be a dense subspace of $X_{\alpha}$. If each product of finitely many $Y_{\alpha}$ has the property that each uncountable subset has an accumulation point, then each continuous function from $X$ to $Z$ depends on countably many coordinates.

Proof. Engelking's proof of [E, Theorem 1] shows that each continuous function from $Y=\prod_{\alpha \in A} Y_{\alpha}$ to $Z$ depends on countably many coordinates. Since $Y$ is dense in $X$, a function on $X$ depends on countably many coordinates if and only if its restriction to $Y$ does, so the conclusion holds.

Notice that if each finite subproduct of $X$ is separable, countably compact or Lindelöf, then $X$ satisfies the hypothesis of the proposition. Under the more restrictive hypothesis on $Z$ that it have a countable base, Ross and Stone show that each continuous function from $X$ to $Z$ depends on countably many coordinates if in $X$ the closure of each open set depends on countably many coordinates. (A set $Y \subset X$ is said to depend on countably many coordinates if for some countable set of coordinates $C$, whenever $y$ belongs to $Y$ and $x \in X$ is any point such that $x_{\alpha}=y_{\alpha}$ for all $\alpha \in C$, then $x$ belongs to $Y$.) Ross and Stone show that the hypothesis on $X$ holds if $X$ satisfies the countable chain condition (although they state a weaker result); the converse holds:

2.2. Proposition. Suppose that, for uncountably many indices $\alpha \in A, X_{\alpha}$ contains a nondense open subset $U_{\alpha}$. Then the closure of each open subset of $X=\prod_{\alpha \in A} X_{\alpha}$ 
depends on countably many coordinates if and only if $X$ satisfies the countable chain condition.

Proof. The "if" direction is shown by the proof of [RS, Theorem 3]. For the other direction, suppose $X$ does not satisfy the countable chain condition. Then, by Corollary 1.4 , we know some finite subproduct fails to satisfy the countable chain condition, so we may suppose that $X$ is $\prod_{\alpha \in \aleph_{1}} X_{\alpha}$ and that $X_{1}$ does not satisfy the countable chain condition. Thus $X_{1}$ contains a family $\left\{V_{\beta}: \beta \in \boldsymbol{\aleph}_{1}\right\}$ of nonempty disjoint open sets. Let $U$ be the union of all sets of the form $V_{\beta} \times U_{\beta}$ $\times \prod_{\alpha \neq 1, \beta} X_{\alpha}$ for $\beta \in \aleph_{1}-\{1\}$. Clearly $U$ is an open subset of $X$. To see that the closure of $U$ does not depend on countably many coordinates, let $C$ be any countable subset of $\boldsymbol{\aleph}_{1}$. Choose any index $\beta \in \aleph_{1}-C$ and let $W_{\beta}$ be a nonempty open subset of $X_{\beta}$ disjoint from $U_{\beta}$. Choose $x \in X$ to be any point such that $x_{1} \in V_{\beta}$ and $x_{\beta} \in U_{\beta}$, and choose $y \in X$ to be any point such that $y_{\alpha}=x_{\alpha}$ for $\alpha \neq \beta$ and $y_{\beta} \in W_{\beta}$. Now, $x \in V_{\beta} \times U_{\beta} \times \prod_{\alpha \neq 1, \beta} X_{\alpha} \subset U$, and $y_{\alpha}=x_{\alpha}$ for all $\alpha \in C$. However, the set $V_{\beta} \times W_{\beta} \times \prod_{\alpha \neq 1, \beta} X_{\alpha}$ is a neighborhood of $y$ which is disjoint from $U$.

Modifying the proof of Ross and Stone it can be shown that each continuous function from $X$ to $Z$ depends on countably many coordinates if $X \times X$ satisfies the countable chain condition and $Z$ has $G_{\delta}^{-}$-diagonal. (The space $Z$ is said to have $G_{\delta}^{-}$-diagonal if the diagonal of $Z \times Z$ can be written as the intersection of countably many of its closed neighborhoods.) Our main result, given below, generalizes this fact.

2.3. THEOREM. Let $X$ be the product of uncountably many nontrivial spaces. Each of the following conditions implies the next, and if $X$ is completely regular, then all are equivalent.

(i) $X$ is pseudo- $\aleph_{1}$-compact.

(ii) For each space $Z$ with $G_{\delta}^{-}$-diagonal, each continuous function from $X$ to $Z$ depends on countably many coordinates.

(iii) Each real-valued continuous function on $X$ depends on countably many coordinates.

Proof. (i) $\Rightarrow$ (ii). Let $f$ be a continuous function from $X$ to $Z$, and let $X^{\prime}$ be a $\Sigma_{0}$-subspace of $X$, say $X^{\prime}=\Sigma_{0}(p)$. Since $X^{\prime}$ is dense in $X$ it suffices to show that the restriction of $f$ to $X^{\prime}$ depends on countably many coordinates. Suppose not. Let $B \subset A$ be maximal with respect to the property that for each index $\beta \in B$ there exist points $x^{\beta}$ and $y^{\beta}$ in $X^{\prime}$ with $x_{\alpha}^{\beta}=y_{\alpha}^{\beta}$ for all $\alpha \neq \beta$, and $f\left(x^{\beta}\right) \neq f\left(y^{\beta}\right)$. Now $B$ must be uncountable. Since $Z$ has $G_{\delta}^{-}$-diagonal, it follows that for some uncountable subset $C$ of $B$ and some closed neighborhood $W$ of the diagonal of $Z \times Z$, the point $\left(f\left(x^{\beta}\right), f\left(y^{\beta}\right)\right)$ is not in $W$ for each $\beta$ in $C$. For each $\beta \in C$ choose basic open neighborhoods $U_{\beta}$ of $x^{\beta}$ and $V_{\beta}$ of $y^{\beta}$ such that $\Pi_{\alpha}\left(U_{\beta}\right)=\Pi_{\alpha}\left(V_{\beta}\right)$ for all $\alpha \neq \beta$ and $f\left(U_{\beta}\right) \times f\left(V_{\beta}\right) \cap W=\varnothing$. 
Since the family $\left\{U_{\beta}: \beta \in C\right\}$ is uncountable, it must have a cluster point $p \in X$. We will show that each basic open neighborhood of $p$ must intersect both $U_{\beta_{0}}$ and $V_{\beta_{0}}$ for some $\beta_{0} \in C$. However, since $W$ is a neighborhood of $(f(p), f(p))$ and $f \times f$ is continuous, this will give the desired contradiction.

Let $O$ be any basic open neighborhood of $p$. Now $O$ must intersect some infinite subfamily $\left\{U_{\beta}: \beta \in C^{\prime}\right\}$ of $\left\{U_{\beta}: \beta \in C\right\}$. Since $R(O)$ is finite we may suppose $R(O) \cap C^{\prime}=\varnothing$. But then, for any $\beta_{0} \in C^{\prime}$, we have $\Pi_{R(O)}\left(U_{\beta_{0}}\right)=\Pi_{R(O)}\left(V_{\beta_{0}}\right)$. Thus $O$ intersects both $U_{\beta_{0}}$ and $V_{\beta_{0}}$.

(ii) $\Rightarrow$ (iii) is trivial. (iii) $\Rightarrow$ (i) for $X$ completely regular: Suppose $X$ is not pseudo- $\aleph_{1}$-compact; then by Corollary 1.5 some finite subproduct of $X$ is not pseudo- $\boldsymbol{N}_{1}$-compact. Thus we may suppose that $X=\prod_{\alpha \in \aleph_{1}} X_{\alpha}$ and that $X_{1}$ is not pseudo- $\boldsymbol{\aleph}_{1}$-compact. That is, $X_{1}$ contains a family $\left\{U_{\alpha}: \alpha \in \boldsymbol{\aleph}_{1}\right\}$ of nonempty disjoint open sets which has no cluster point. For each $\alpha$ let $g_{\alpha}$ be a nonconstant continuous real-valued function on $X_{1}$ which is zero on the complement of $U_{\alpha}$ and let $h_{\alpha}$ be a nonconstant continuous real-valued function on $X_{\alpha}$. The function defined by the rule $f(x)=\sum_{\alpha \in \mathbb{N}_{1}} g_{\alpha}\left(x_{1}\right) h_{\alpha}\left(x_{\alpha}\right)$ is continuous and does not depend on countably many coordinates, so we have the desired contradiction.

Regarding the condition that $Z$ have a $G_{\delta}^{-}$-diagonal, note that every submetrizable space has this property. (A space is said to be submetrizable provided some continuous one-to-one image is metrizable.) The converse is true if $Z$ is countably compact (in this case $Z$ is metrizable) $\left[\mathrm{A}_{1}\right]$ or if $Z$ is paracompact and has a $G_{\delta^{-}}$ diagonal $[\mathrm{K}]$. Also, it is shown in $[\mathrm{K}]$ that each regular developable space has $G_{\delta}^{-}$-diagonal. Of course, if $Z \times Z$ is normal, then $Z$ has a $G_{\delta}^{-}$-diagonal if and only if it has a $G_{\delta}$-diagonal.

2.4. CoROllary to PROOF. Let $X$ be pseudo- $\aleph_{1}$-compact, let $Y$ be a subspace of $X$ and suppose $Z$ has $G_{\delta}^{-}$-diagonal. If $Y$ contains a $\Sigma_{0}$-subspace of $X$, then each continuous function from $Y$ to $Z$ depends on countably many coordinates.

Following Corson $\left[\mathrm{C}_{3}\right]$, we call a subspace of a product space $X=\prod_{\alpha \in A} X_{\alpha}$ a $\Sigma$-subspace if it has the form $\left\{x \in X: x_{\alpha} \neq p_{\alpha}\right.$ for at most countably many $\left.\alpha \in A\right\}$ for some point $p \in X$. Note that if $X^{\prime}$ is a $\Sigma$-subspace of $X$ and some continuous function $f$ on $X^{\prime}$ depends on countably many coordinates, then $f$ has a continuous extension to all of $X$. Thus we have the following corollary:

2.5. Corollary. If $X$ is pseudo- $\aleph_{1}$-compact and $Y$ contains a $\Sigma$-subspace of $X$, then $Y$ is $C$-embedded in $X$.

If $X$ is realcompact, it follows that $X=v Y$, where $v Y$ denotes the Hewitt realcompactification of $Y$. For some additional conditions under which $\Sigma$-subspaces will be $C$-embedded, see [ $\left.\mathrm{U}_{1}\right]$.

It has been called to our attention that Comfort and Negrepontis [CN] have been able to achieve the following result: 
2.6. THEOREM. Let $X$ be the product of completely regular Hausdorff spaces. If $Y$ is dense in $X$ and is pseudo- $\aleph_{1}$-compact, then each continuous real-valued function on $Y$ depends on countably many coordinates.

3. Analog for higher cardinals. Contrary to the heading, we begin by considering the analog for lower cardinals. Recall that spaces which are otherwise quite reasonable can have the property that each continuous real-valued function is constant $[\mathrm{H}],\left[\mathrm{A}_{2}\right]$, and $[\mathrm{Y}]$.

3.1. Proposition. Each continuous real-valued function on a product space $X$ depends on finitely many coordinates if and only if all but finitely many of the factors have the property that each real-valued continuous function is constant.

Proof. If for each integer $n$ there exist an index $\alpha_{n}$ and a nonconstant continuous function $f_{n}: X_{\alpha_{n}} \rightarrow I_{n}$, for $I_{n}=\left[0,1 / 2^{n}\right]$, then the function $f(x)=\sum_{n \in N} f_{n}\left(x_{\alpha_{n}}\right)$ is a continuous function which does not depend on finitely many coordinates. The converse is obvious.

In adapting the proof of Theorem 2.3 to the case of higher cardinals the only difficulty comes in showing that the set of coordinates $B$ (where for each index $\beta \in B$ the points $x^{\beta}$ and $y^{\beta}$ have been chosen so that $\left.f\left(x^{\beta}\right) \neq f\left(y^{\beta}\right)\right)$ contains a subset $C$ with card $(C)=$ card $(B)$ such that, for some closed neighborhood $W$ of the diagonal, $\left(f\left(x^{\beta}\right), f\left(y^{\beta}\right)\right)$ is not in $W$ for each $\beta$ in $C$. Since the diagonal is the intersection of countably many closed neighborhoods, this can be done if the cardinality of $B$ is not the supremum of countably many smaller cardinals. Thus

3.2. Theorem. Let $X$ be the product of at least $\boldsymbol{\aleph}$ nontrivial spaces, and let $Y$ be a subspace which contains a $\Sigma_{0}$-subspace. If $\aleph$ is not the supremum of countably many smaller cardinals, then each of the following conditions implies the next, and if $Y$ is completely regular, then all are equivalent.

(i) The space $Y$ is pseudo-א-compact.

(ii) For each space $Z$ with $G_{\delta}^{-}$-diagonal, each continuous function from $Y$ to $Z$ depends on fewer than $\boldsymbol{\aleph}$ coordinates.

(iii) Each real-valued continuous function on $Y$ depends on fewer than $\boldsymbol{\aleph}$ coordinates.

Proof. This follows by a straightforward adaptation of the proof of Theorem 2.3.

Our next result gives conditions under which (i) of Theorem 3.2 will hold. For this we generalize the notion of a $\Sigma_{0}$-subspace. For an infinite cardinal $\boldsymbol{\aleph}_{\gamma}$ and a point $p$ in $X$, let $\Sigma_{\gamma}(p)=\left\{x \in X:\right.$ card $\left.\left(\left\{\alpha: x_{\alpha} \neq p_{\alpha}\right\}\right)<\boldsymbol{N}_{\gamma}\right\}$. We call such sets $\Sigma_{\gamma}$-subspaces.

3.3. Proposition. Let $\boldsymbol{\aleph}$ be a cardinal with cof $(\boldsymbol{\aleph})=\boldsymbol{\aleph}_{\gamma}>\boldsymbol{\aleph}_{0}$, and let $X$ be a product space. A subspace $Y$ of $X$ is pseudo-א-compact under either of the following two conditions.

(i) $\boldsymbol{N}$ is regular, $Y$ contains a $\Sigma_{0}$-subspace, and each finite subproduct of $X$ is pseudo-א-compact. 
(ii) $\boldsymbol{\aleph}$ is singular, $Y$ contains a $\Sigma_{\gamma+1}$-subspace, and each $\boldsymbol{\aleph}_{\gamma}$-fold subproduct of $X$ is pseudo-א-compact.

Proof. Since a space which contains a dense pseudo- $\boldsymbol{N}$-compact subspace is itself pseudo- $\boldsymbol{\kappa}$-compact, we may suppose that $Y$ is itself a $\Sigma_{\delta}$-subspace, for $\delta=0$ in case (i) and $\delta=\gamma+1$ in case (ii). A straightforward generalization of the proof of Theorem 1.3 shows that such subspaces are pseudo- $\boldsymbol{\aleph}$-compact.

Our final result shows that the requirement that $\boldsymbol{\aleph}$ not be the supremum of a countable set of smaller cardinals cannot be weakened.

3.4. Proposition. Let $\aleph$ be a cardinal which is the supremum of countably many smaller cardinals, and let $X$ be a completely regular product with at least $\boldsymbol{\aleph}$ nontrivial factors. If for each cardinal $m$ less than $\mathbb{N}, X$ is not pseudo-m-compact, then there exists a continuous real-valued function on $X$ which does not depend on fewer than $\boldsymbol{N}$ coordinates.

Proof. Suppose $\boldsymbol{\aleph}=\sup _{n \in N} \boldsymbol{\aleph}_{\gamma_{n}}$, where each $\boldsymbol{\aleph}_{\gamma_{n}}$ is chosen to be regular and uncountable. By Theorem 3.2, there exists for each integer $n$ a continuous realvalued function $f_{n}$ which depends on $\boldsymbol{\aleph}_{\gamma_{n}}$ coordinates. Furthermore, we can choose these functions so that for each $n$ the range of $f_{n}$ is precisely $\left[0,1 / 2^{n}\right]$. The function $f(x)=\sum_{n \in N} f_{n}(x)$ is continuous and depends on $\boldsymbol{\aleph}$ coordinates.

As a final area of generalization, it is worth noting that if the diagonal in $Z \times Z$ can be written as the intersection of $m$ closed neighborhoods, then each continuous function from $X$ to $Z$ depends on fewer than $\boldsymbol{N}$ coordinates provided $X$ is pseudo$\boldsymbol{\aleph}$-compact and cof $(\boldsymbol{N})<\mathfrak{m}$. The proof of this is a straightforward adaptation of the proof of Theorem 2.3.

\section{REFERENCES}

[A ]. B. A. Anderson, Topologies comparable to metric topologies, Topology Conference, Arizona State University, Tempe, Ariz., 1967, pp. 15-21.

$\left[\mathrm{A}_{2}\right]$. S. Armentrout, $A$ Moore space on which every real-valued continuous function is constant, Proc. Amer. Math. Soc. 12 (1961), 106-109. MR 22 \#11365.

$\left[\mathrm{C}_{1}\right]$. W. W. Comfort, A nonpseudocompact product space whose finite subproducts are pseudocompact, Math. Ann. 170 (1967), 41-44. MR 35 \#965.

$\left[\mathrm{C}_{2}\right]$. - Theory of cardinal invariants, General Topology and its Applications, SpringerVerlag (to appear).

$\left[\mathrm{C}_{3}\right]$. H. H. Corson, Normality in subsets of product spaces, Amer. J. Math. 81 (1959), 785-796. MR 21 \#5947.

[CN]. W. W. Comfort and S. Negrepontis, Ultrafilters and the Stone-Čech compactification (to appear).

[D]. R. O. Davies, An intersection theorem of Erdös and Rado, Proc. Cambridge Philos. Soc. 63 (1967), 995-996. MR 35 \#6570.

[E]. R. Engelking, On functions defined on Cartesian products, Fund. Math. 59 (1966), 221-231. MR 34 \#3546.

[ER]. P. Erdös and R. Rado, Intersection theorems for systems of sets, J. London Math. Soc. 35 (1960), 85-90. MR 22 \#2554.

[F]. Z. Frolík, On two problems of W.W. Comfort, Comment. Math. Univ. Carolinae 8 (1967), 139-144. MR 35 \#966. 
[G]. I. Glicksberg, Stone-Čech compactifications of products, Trans. Amer. Math. Soc. 90 (1959), 369-382. MR 21 \#4405.

[GJ]. L. Gillman and M. Jerison, Rings of continuous functions, University Series in Higher Math., Van Nostrand, Princeton, N. J., 1960. MR 22 \#6994.

[H]. E. Hewitt, On two problems of Urysohn, Ann. of Math. (2) 47 (1946), 503-509. MR 8, 165.

[K]. D. Kullman, A note on developable spaces and p-spaces (to appear).

$\left[\mathrm{M}_{1}\right]$. E. Marczewski, Séparabilité et multiplication cartésienne des espaces topologiques, Fund. Math. 34 (1947), 127-143. MR 9, 98.

$\left[\mathrm{M}_{2}\right]$. R. H. Marty, Mazur theorem and m-adic spaces, Doctoral Dissertation, Pennsylvania State University, University Park, Pa., 1969.

$\left[\mathrm{M}_{3}\right]$. S. Mazur, On continuous mappings on Cartesian products, Fund. Math. 39 (1953), 229-238. MR 14, 1107.

[M4]. E. Michael, A note on intersections, Proc. Amer. Math. Soc. 13 (1962), 281-283. MR 24 \#A3070.

$\left[\mathrm{M}_{5}\right]$. A. Miščenko, Several theorems on products of topological spaces, Fund. Math. 58 (1966), 259-284. (Russian) MR 33 \#4884a.

[RS]. K. A. Ross and A. H. Stone, Products of separable spaces, Amer. Math. Monthly 71 (1964), 398-403. MR 29 \#1611.

$\left[\mathrm{S}_{1}\right]$. N. A. Sanin, $A$ theorem from the general theory of sets, C. R. (Dokl.) Acad. Sci. URSS 53 (1946), 399-400. MR 8, 333.

$\left[\mathrm{S}_{2}\right]$. - On intersection of open subsets in the product of topological spaces, C. R. (Dokl.) Acad. Sci. URSS 53 (1946), 499-501. MR 8, 334.

$\left[\mathrm{S}_{3}\right] .-$ On the product of topological spaces, Trudy Mat. Inst. Steklov. 24 (1948), 112 pp. (Russian) MR 10, 287.

[U $\mathrm{U}_{1}$ ]. M. Ulmer, C-embedded II-spaces, Notices Amer. Math. Soc. 16 (1969), 849. Abstract \#69T-G105.

$\left[\mathrm{U}_{2}\right] .-$, Continuous functions on product spaces, Doctoral Dissertation, Wesleyan University, Middletown, Conn., 1970.

$\left[\mathrm{U}_{3}\right]$. - Functions on product spaces, Notices Amer. Math. Soc. 16 (1969), 986-987. Abstract \#69T-G134.

$\left[\mathrm{U}_{4}\right] .-$, The countable chain condition, Notices Amer. Math. Soc. 17 (1970), 462-463. Abstract \#70T-G24.

[V]. G. Vidossich, Two remarks on A. Gleason's factorization theorem, Bull. Amer. Math. Soc. 76 (1970), 370-371. MR 41 \#1021.

[Y]. J. N. Younglove, A locally connected, complete Moore space on which every real-valued continuous function is constant, Proc. Amer. Math. Soc. 20 (1969), 527-530. MR 40 \#1992.

Canary Road, Westlake, Oregon 97493

Department of Mathematics, Macalester College, Saint Paul, Minnesota 55101 